\title{
The influence of inhomogeneities on hot star wind model predictions
}

\author{
J. Krtička ${ }^{1}$, L. Muijres ${ }^{2}$, J. Puls $^{3}$, J. Kubát ${ }^{4}$ and A. de Koter ${ }^{2}$ \\ ${ }^{1}$ Institute of Theoretical Physics and Astrophysics, Masaryk University, Brno, Czech Republic \\ ${ }^{2}$ Astronomical Institute, "Anton Pannekoek", University of Amsterdam, Amsterdam, \\ The Netherlands \\ ${ }^{3}$ Universitätssternwarte München, München, Germany \\ ${ }^{4}$ Astronomický ústav, Ondřejov, Czech Republic
}

\begin{abstract}
We study the effect of wind inhomogeneities (clumping) on $\mathrm{O}$ star wind model predictions. For this purpose we artificially include clumping into our stationary NLTE wind models. As a result of the inclusion of optically thin clumps the radiative line force is increased compared to corresponding unclumped models, with a similar effect on either the mass-loss rate or the terminal velocity. When the clumps are allowed to be optically thick in continuum, on the other hand, the radiative force and consequently the mass-loss rate decreases alternatively.
\end{abstract}

Keywords. Stars: mass loss, stars: early-type, hydrodynamics

\section{Introduction}

From the beginning of the quantitative studies of the stellar wind of hot stars it seemed clear that the mass-loss should significantly influence the evolution of these stars. The evolution of massive stars from the main sequence to the final supernova explosion was considered to be driven mainly by the intense winds of these stars. The classical studies of hot star winds culminated by the application of NLTE models both for the determination of hot star wind properties from the observational data (Puls et al. 1996) and for the prediction of wind mass-loss rates (Vink et al. 2000, Pauldrach et al. 2001, Krtička \& Kubát 2004). These models generally assumed that the stellar wind can be described in a first approximation by the smooth, basically spherically symmetric outflow. Generally, there was a satisfactory agreement between theoretical predictions and observations.

The picture of smooth outflow was known to be inadequate due to the existence of the inherent instability connected with the radiative driving (Owocki \& Rybicki 1984, Feldmeier et al. 1997). From the observational point of view, the existence of wind inhomogeneities (or wind "clumping") is not so apparent. The reason is that most diagnostical features depend on the product $\sqrt{C_{\mathrm{c}}} \dot{M}$, where the "clumping factor" $C_{\mathrm{c}} \geqslant 1$ relates the density inside the clump $\rho^{+}$with the mean wind density $\langle\rho\rangle$,

$$
\rho^{+}=C_{\mathrm{c}}\langle\rho\rangle \text {. }
$$

Consequently, spectra from winds with a large clumping factor but small mass-loss rate can mimic those from winds with weak clumping but large mass-loss rate. If $C_{\mathrm{c}}>1$, then the mass-loss rates derived from such diagnostics are overestimated by factor of $\sqrt{C_{\mathrm{c}}}$. Fortunately, some spectral properties may be used to break this degeneracy, and to estimate the value of $C_{\mathrm{c}}$. For example, Martins et al. (2005b) derived clumping a factor of $10-100$ for studied Galactic O-stars. If these values of clumping factor are real, this would imply a decrease of the estimated mass-loss rate by factors of 3-10. 


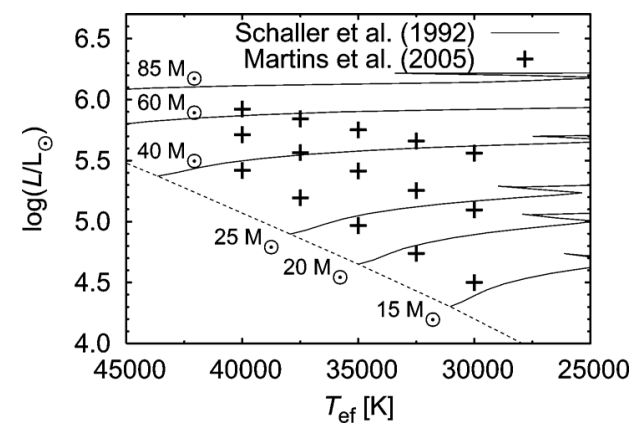

Figure 1. Parameters of studied stars in the HR diagram. Overplotted are the evolutionary tracks from Schaller et al. (1992)

Stimulated by these studies, we discuss here the influence of inhomogeneities on the mass-loss rate predictions.

\section{Wind models}

For our study we used the spherically symmetric, stationary NLTE wind models (Krtička \& Kubát 2004). These models solve the equations of statistical equilibrium together with the equations of radiative transfer. The calculated occupation numbers are used to derive the radiative force (in the Sobolev approximation) and the radiative heating/cooling terms. This enables us to obtain the radial stratification of velocity, density and temperature in the wind and finally to predict the wind mass-loss rate $\dot{M}$ and terminal velocity $v_{\infty}$.

The wind models are calculated for O-type stellar parameters based on the recent calibrations by Martins et al. (2005a, see also Fig. 1).

\section{Influence on statistical equilibrium equations: clumping}

Wind inhomogeneities may influence the statistical equilibrium equations due to change of the electron density. This effect can be roughly accounted for within the clumping approximation, which is widely used to study the wind spectra, e.g., Martins et al. (2005b).

To include the effect of clumping into our models we modified the equations of statistical equilibrium, by using an electron density $\rho_{\mathrm{e}}^{+}=C_{\mathrm{c}}\left\langle\rho_{\mathrm{e}}\right\rangle$, opacity $\langle\chi\rangle=\chi^{+} / C_{\mathrm{c}}$, and emissivity $\langle\eta\rangle=\eta^{+} / C_{\mathrm{c}}$. The superscript + denotes values inside the (homogeneous) clumps and the quantities inside brackets corresponding volume averages.

\subsection{Influence of clumping}

To investigate the influence of clumping on the stellar wind we have calculated a wind model of an O-type giant at $T_{\text {eff }}=35000 \mathrm{~K}$, assuming the wind to be smooth $\left(C_{\mathrm{c}}=1\right)$ close to the star $\left(r<2 R_{*}\right)$, and to be clumped $\left(C_{\mathrm{c}}=10\right)$ in the outer regions $\left(r>2 R_{*}\right)$.

In accord with our assumptions, the presence of clumping leads to an increase of the electron density inside the clumps. Consequently, the recombination rates become higher and the wind becomes less ionized (see Fig. 2). Since lower ions are able to accelerate the stellar wind more efficiently than the higher ones (due to a larger number of driving lines), the radiative force increases, which, in our case, leads to an increase in wind velocity. 

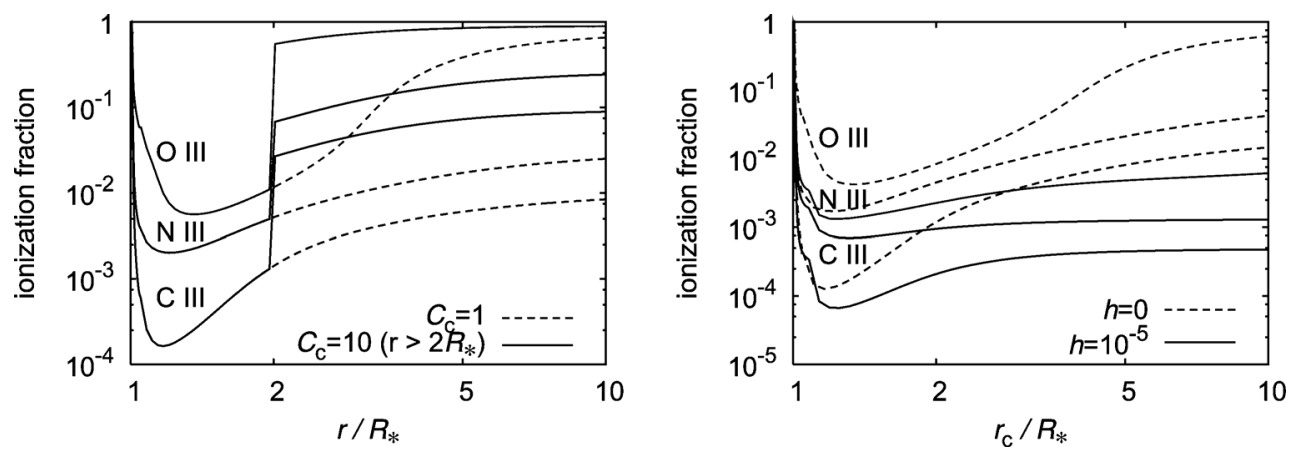

Figure 2. Left: Influence of clumping that starts at $r=2 R_{*}$ on the ionization fractions of selected ions. Right: Influence of porosity on the ionization fractions.

\subsection{Radially constant clumping factor}

The influence of clumping on the wind parameters depends on the radial onset of clumping. If clumping starts above the critical point (below which the mass-loss rate is determined), then the terminal velocity increases. On the other hand, if clumping starts below the critical point, then the wind mass-loss rate becomes larger.

In case of radially constant clumping factor, the mass-loss rate increases significantly (on average by a factor of 2 for $C_{\mathrm{c}}=10$ ), and the predicted wind-momentum is also higher than for a smooth wind at the same parameters (see Fig. 3).

\section{Porosity}

Wind inhomogeneities may also influence the wind radiative transfer if the individual clumps are optically thin in continuum. This effect is termed as a porosity (Owocki et al. 2004), and can be introduced into the wind models by decreasing the continuum opacity,

$$
\chi_{\mathrm{eff}}=\frac{\langle\chi\rangle}{1+r h\langle\chi\rangle},
$$

where $h r$ is the porosity length. The continuum emissivity has to be modified by the same factor.

The porosity leads to a significant increase of the wind ionization (see Fig. 2 for the results calculated for $C_{\mathrm{c}}=10$, and $h=0$ and $h=10^{-5}$ ). The increased ionization causes a decrease of the radiative force, and also of the mass-loss rate if the wind is porous below the critical point (see Fig. 3 for the results for radially constant $C_{\mathrm{c}}$ and $h$ ).

\section{Influence on the radiative force}

Up to now we discussed indirect influence of inhomogeneities on the line radiative force caused by the change of the wind ionization. As the radiative force depends on the velocity via the Doppler effect, the presence of inhomogeneities may influence the radiative force also directly. However, numerical simulations (Owocki \& Rybicki 1984, Feldmeier et al. 1997) show that on average the instabilities do not directly affect the line radiative force. Consequently, we neglected the direct influence of inhomogeneities on the line radiative force. 


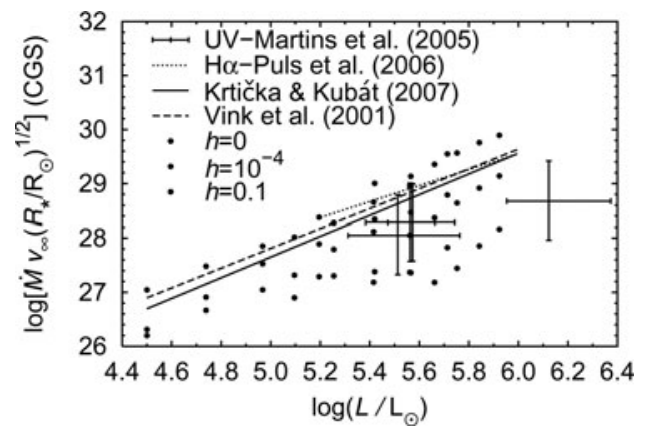

Figure 3. Influence of wind inhomogeneities on the modified wind-momentum (for $C_{\mathrm{c}}=10$ ).

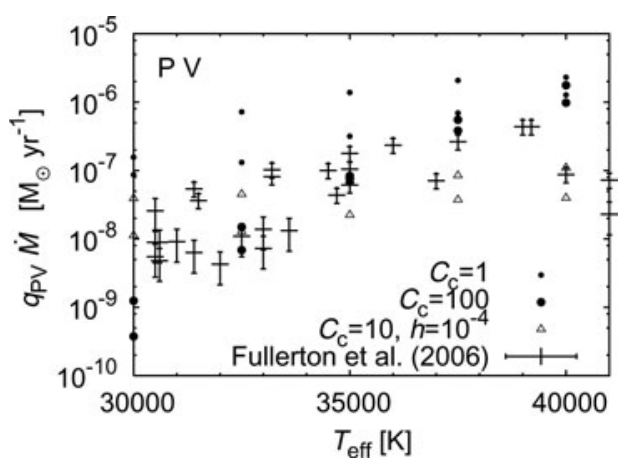

Figure 4. Influence of different types of wind inhomogeneities on the P V ionization.

\section{Discussion}

We conclude that wind inhomogeneities may influence the predicted wind parameters. An approach which roughly corresponds to the results of time-dependent simulations (i.e., inhomogeneities that "start" above the critical point) does not improve the agreement between theory and observations. On the other hand, if the inhomogeneities start below the critical point, then the effects of clumping and porosity may provide a better agreement between theory and observations, both in terms of modified wind momentum (Fig. 3) and P V ionization fractions (Fig. 4).

\section{Acknowledgements}

This research was supported by grants grants GA ČR 205/07/0031, and 205/08/0003.

\section{References}

Feldmeier, A., Puls, J., \& Pauldrach, A. W. A. 1997, A\&3A 322, 878

Krtička, J. \& Kubát, J. 2004, A\&A 417, 1003

Martins, F., Schaerer, D., \& Hillier, D. J. 2005a, A\&SA 436, 1049

Martins F., Schaerer, D., Hillier, D. J., et al. 2005b, $A \& A$ 441, 735

Owocki, S. P., Gayley, K. G., \& Shaviv, N. J. 2004, ApJ 616, 525

Owocki, S. P. \& Rybicki, G. B. 1984, ApJ 284, 337

Pauldrach, A. W. A., Hoffmann, T. L., \& Lennon, M. 2001, A\&A 375, 161

Puls, J., Kudritzki, R.-P., et al. 1996, A\&\&A 305, 171

Schaller, G., Schaerer, D., Meynet, G., \& Maeder, A. 1992, A\&AS 96, 269

Vink, J. S., de Koter, A., \& Lamers, H. J. G. L. M., 2000, A $\& A$ 362, 295 


\section{Discussion}

WoITKE: What confines your "clumps"? In reality, there will be rather turbulence leading to filaments, that could be mistaken for "clumps".

KRTIČKA: The process of radiative acceleration has been shown to cause instabilities, which is different from "normal" turbulence. Need 3D hydro with line transfer to reveal the nature of these structures. 